\title{
Length of Stay in a Community Psychiatric Emergency Room: An Analysis of Contributing Factors
}

\author{
Chiedozie Ojimba *, M.D., Adenike Ishola, M.D., Oluwole Jegede M.D., Terence Tumenta, M.D., Samuel Adeyemo, \\ M.D., Olaniyi Olayinka, M.D., Alexander Maksymenko, M.D., Susmita Khadka, M.D., Andrew Shapiro, Karthik \\ Cherukupally, M.D., Ayotomide Oyelakin, M.D., Tolu Olupona, M.D., Jason Hershberger, M.D.,
}

Interfaith Medical Center, Brooklyn, New York

Correspondence to: Chiedozie Ojimba MD, MPH; cojimba@interfaithmedical.org

Received 07 August 2020;

Accepted 20 August 2020;

Published 01 September 2020

\begin{abstract}
Background: The length of patients' stay in the emergency room is a key measure of service delivery and a marker to measure the quality of care. Studies have shown that patients with psychiatric and substance use disorders have a prolonged length of stay in the emergency room compared to medical and surgical patients. Various factors have been found to contribute to this disparity. Method: This is a retrospective case by case review of four hundred and ninety-three consecutive patients who presented to a community psychiatric emergency. Results: Our results show a length of stay ranging between $1.21-33.48$ hours. The mean length of stay of 8.72 hours and the median was 7.41 hours. Furthermore, the utilization of emergency medication and age above 44 have a significant influence on patients' length of stay in the psychiatric emergency room ( $\mathrm{p}<0.05$ ). Conclusion: The length of stay in the psychiatric emergency room deserves more study in literature as it remains a metric for service delivery. Although, factors contributing to a prolonged length of stay may vary, in different settings and situations, understanding these factors will improve outcomes for patients in psychiatric emergency rooms.
\end{abstract}

Keywords: Length of stay, psychiatry emergency department, detoxification, Inpatient, substance use and medicating for aggression.

\section{Introduction}

The emergency department (ED) is an essential part of psychiatric service but the hospitals have limited capacity for ED psychiatric care ${ }^{[1]}$. According to the United States Agency for Healthcare Research and Quality (AHRQ), about one in eight ED visits in the United States is for treatment of mental health or substance use disorders (M/SUDs) ${ }^{[1]}$. Between 2007 and 2011, the rate of ED visits related to M/SUDs increased by over 15 percent ${ }^{[2]}$. Another study showed the rate of ED visits per 100,000 population related to M/SUDs increased substantially between 2006 and 2013. The increase over these 7 years was higher for mental disorders (55.5 percent for depression, anxiety or stress reactions and 52.0 percent for psychoses or bipolar disorders) than for substance use disorders (37.0 percent) ${ }^{[3]}$.

Length of stay (LOS) is a key measure of ED throughput and a marker of overcrowding ${ }^{[4]}$. ED crowding manifests as long waits for patients to be seen by providers, high left-without-beingseen rates, long ED lengths of stay (LOS), and long waiting times before inpatient bed placement (also known as ED boarding time)

${ }^{[5]}$. Time studies that assess key ED processes will help clarify the causes of patient care delays and prolonged LOS ${ }^{[6]}$. Psychiatric patients remain in the ED far longer than medical patients as boarding of psychiatric patients in the ED may range from hours to days. The average ED LOS for psychiatric patients ranges from 7 to 34 hours in the United States. Other studies have also reported that the length of stay for psychiatric patients in the ED is in excesses of 21 hours and as high as 48 hours ${ }^{[7-9]}$. Furthermore, patients experiencing mental health emergencies are reported to wait three times as long for inpatient beds than those with other medical emergencies ${ }^{[7]}$. A 2008 American College of Emergency Physicians (ACEP) survey of 1400 ED directors revealed $79 \%$ of the respondents reported psychiatric emergencies board in their ED daily ${ }^{[10]}$ and patients presenting to the ED for mental health crises are 2.5 times more likely to need to be admitted ${ }^{[7]}$.

Factors that influence patients' length of stay include patients' demographic characteristics of age and ethnicity, presence of junior residents or medical students increased testing, consultation, and radiology studies ${ }^{[8]}$. Potential adverse effects of prolonged ED boarding times on patient quality of care, hospital operations, and financial outcomes have also been reported ${ }^{[11]}$. Such adverse effects include several negative patient-oriented outcomes and high inpatient mortality rates ${ }^{[11]}$. Reducing the 
length of stay could decrease psychological stress on patients and speed up mental health treatment that could mitigate the need for a mental health inpatient stay and overall outcome. Liew and Kennedy reported that patients who remain in the ED for up to 8 hours are $20 \%$ more likely to stay in hospital longer than the national average for the relevant admission problem ${ }^{[12]}$.

The objective of this study is to determine which factors contribute to prolonged patients' length of stay (LOS) in our psychiatric emergency room.

\section{Methods}

\section{Study setting and population}

This study is a retrospective study conducted at the psychiatry emergency department of a community teaching hospital, in Brooklyn, New York. The hospital has a psychiatric emergency room, 90 inpatient psychiatry beds, 20 inpatient detoxification beds, and 20 inpatient rehabilitation beds. The hospital serves a predominantly African American population. The hospital where this study is conducted serves a predominantly African American population. It provides around-the-clock, year-round emergency psychiatric care. The main treatment goals of the psychiatric emergency department (PED) are to provide effective assessment, rapid stabilization, and appropriate placement of patients. It is equipped with a staff composed of an attending psychiatrist, a resident psychiatrist, psychiatric nurse practitioner, and nurses always. Substance use assessment coordinator is also available during standard working hours to provide necessary assistance in screening patients who presented for detoxification program. The annual census is approximately 6000 visits.

\section{Study design}

The investigators manually reviewed the electronic medical records (EMR) of all patients who presented to the psychiatric ED between midnight December 1, 2017, and midnight December 31, 2017 and were admitted to inpatient units (psychiatry and detox). A sample of 493 visits was generated using the sampling analysis tool in Microsoft Excel. The variables retrieved from the EMR included: patients' age, gender, race, marital status, living arrangement, substance use, date and time of arrival to the hospital, arrival time to the PED, time evaluated by the psychiatrist, whether a patient was medicated due to aggression or psychotic agitation, medical clearance time, departure time to inpatient or detoxification unit, and the shift schedule during which the patients were evaluated. Total LOS was calculated by adding the time spent in the medical ED and the PED. LOS was defined as the time from arrival to the hospital to leaving the emergency room to inpatient units. There is no universally agreed timeline for treating mentally ill patients in the United State of America (USA). The Joint Commission recognizes that specific boarding timeframes will vary from one organization to the other; for this reason, the 4-hour time frame recommended by Joint Commission for medical and surgical patients; 6 hours for psychiatry patients serves as a guideline to help set a reasonable goal for institutions. For the purpose of our study, we excluded patients treated and released from the PED and patients transferred to the medical ED or medical floors for medical stabilization.

\section{Statistical analysis}

Statistical analysis was performed with STATA 16 software for windows. A total of ten variables (1 numerical and 9 categorical) were included for the baseline analysis and data description. Univariate frequencies and summary statistics were used to describe the study population and summarize the response and independent variables. Odds ratios (OR) were determined by logistic regression analysis of bivariate and multivariate analyses. A p-value of less than 0.05 was considered statistically significant for all analyses in the study and all p-values were reported as twotailed. All variables in the bivariate analysis were included in the multivariate analysis. The variables race and marital status with 3 or more categories were entered in the model in the form of dummy variables. Accordingly, multivariate analysis was done adjusting simultaneously for age, race, sex, marital status, living arrangement, emergency medication, work shift, and admission disposition.

The Hosmer-Lemeshow and Pearson test were used to check the goodness-of-fit of the model. The lack of statistical significance of the goodness-of-fit model provides enough assurance that the logistic regression model we used fits our observations.

This study was reviewed and approved by the Ethics Committee, Institutional Review Board (IRB) of Interfaith Medical Center (IMC), and the requirement for informed consent was waived by the Ethics Committee.

\section{Results}

There were 493 patients that presented the emergency department during the study month in review, of this number, 221 patients were seen, treated, and discharged from the ED. Of the 250 patients who met criteria for inpatient admission, 150 (60.0\%) were admitted to the inpatient psychiatric unit and 100 (40.0\%) were admitted to the substance use detoxification unit, and all were included in analysis (Table 1). Twenty-two (22) patients who were originally cleared by the psychiatrist for inpatient admission failed medical clearance and had to be transferred to the medical inpatient unit or back to the medical emergency department. Of the 250 patients, 71.6\% were African America, 11.65 were White, $13.2 \%$ reported being Hispanic, and 3.6 were identified as other race. Further, $71.6 \%$ of the participants were male and $28.4 \%$ identified as female. The median age of the study patients was 44 years old, with the youngest being 19 years old and the oldest 90 years old. The mean was also 44 years with a standard deviation of 13 . The length of stay ranged from 73 minutes (1.21 hours) to 2009 minutes (33.48 hours). The mean length of stay of 8.72 hours and the median was 7.41 hours. Due to the skewness of the distribution, we used the median length of stay for our analysis.

In bivariate analyses (Table 2), being older ( $\geq$ 44years) [OR: 2.41, 95\% Confidence Interval (CI): 1.45-3.99] and receiving IM medication [OR: 2.08; 95\% Confidence Interval (CI): 1.123.86] were significantly associated with a longer length of stay, with p-values of 0.001 and 0.020 respectively. American Americans compared to Hispanics [OR:1.59], evaluation during a weekday shift [OR:1.25], and admission to the inpatient psychiatry unit [OR:1.36] appear to have an impact on prolonging the length of stay, though they were not statistically in our study.

In multivariate analysis, where all covariates in the unadjusted model were included, only variable age group (Older adults vs young adults) was significantly associated with a longer length of stay (Table 3).

The results from the Pearson goodness of fit (number of groups $=95 ; \chi 2=84.06 ; \quad$-value $=0.48)$ and Hosmer-Lemeshow good of fit (number of groups $=9 ; \chi 2=3.66 ; p$-value $=0.81$ ) indicate that the fitted model cannot be rejected and thus leads to the conclusion that the model fits well (Table 4). 
Table 1: Characteristics of Baseline Study Population by Length of Stay Category (LOS)

\begin{tabular}{|c|c|c|c|}
\hline Variables & $\begin{array}{l}\text { LOS }<7.41 H \\
(49.6 \%, n=124)\end{array}$ & $\begin{array}{l}\text { LOS }>7.41 H \\
(50.4 \%, n=126)\end{array}$ & $\begin{array}{l}\text { Total } \\
(100 \%, n=250)\end{array}$ \\
\hline Median age (IQR, SD) ${ }^{\text {a }}$ & $39.5(30.5-51,12.4)$ & $48.5(36-55,13.3)$ & $44(33-54,13.2)$ \\
\hline \multicolumn{4}{|l|}{$\operatorname{Age} \operatorname{Group}(\%, \mathbf{n})^{*}$} \\
\hline Young adults (19-44) & $60.5(75)$ & $38.9(49)$ & $49.6(124)$ \\
\hline Older adults $(\geq 44)$ & $39.5(49)$ & $61.1(77)$ & $50.4(126)$ \\
\hline \multicolumn{4}{|l|}{ Gender (n, \%) } \\
\hline Male & $70.2(87)$ & $73.0(92)$ & $71.6(179)$ \\
\hline Female & $29.8(37)$ & $27.0(34)$ & $28.4(71)$ \\
\hline \multicolumn{4}{|l|}{ Race $(\%, \mathbf{n})$} \\
\hline African American & $73.4(91)$ & $69.8(88)$ & $71.6(179)$ \\
\hline White & $12.1(15)$ & $11.1(14)$ & $11.6(29)$ \\
\hline Hispanic & $10.5(13$ & $15.9(20)$ & $13.2(33)$ \\
\hline Others & $4.0(5)$ & $3.2(4)$ & $3.6(9)$ \\
\hline \multicolumn{4}{|l|}{ Marital Status $(\%, \mathbf{n})$} \\
\hline Single & $90.3(112)$ & $92.0(116)$ & $91.2(228)$ \\
\hline Married & $4.8(6)$ & $4.0(5)$ & $4.4(11)$ \\
\hline Unknown & $4.8(6)$ & $4.0(5)$ & $4.4(11)$ \\
\hline \multicolumn{4}{|c|}{ Living Arrangement $(\%, n)$} \\
\hline Homeless & $17.7(22)$ & $19.8(25)$ & $18.8(47)$ \\
\hline Domiciled & $82.3(102)$ & $80.2(101)$ & $81.2(203)$ \\
\hline \multicolumn{4}{|l|}{ Substance Use $(\%, n)$} \\
\hline No & $19.3(24)$ & $19.8(25)$ & $19.6(49)$ \\
\hline Yes & $73.4(91)$ & $73.8(93)$ & $73.6(184)$ \\
\hline Unknown & $7.3(9)$ & $6.4(8)$ & $6.8(17)$ \\
\hline \multicolumn{4}{|l|}{ IM Medication (\%, n) } \\
\hline No & $71.8(89)$ & $84.1(106)$ & $78.0(195)$ \\
\hline Yes & $28.2(35)$ & $15.9(20)$ & $22.0(55)$ \\
\hline \multicolumn{4}{|l|}{ Shift $(\%, n)$} \\
\hline Weekday & $75.8(94)$ & $71.4(90)$ & $73.6(184)$ \\
\hline Weekend & $24.2(30)$ & $28.6(36)$ & $26.4(66)$ \\
\hline \multicolumn{4}{|c|}{ Admission disposition $(\%, \mathbf{n})$} \\
\hline Inpatient unit & $63.7(79)$ & $56.3(71)$ & $60.0(150)$ \\
\hline Detox unit & $36.3(45)$ & $43.7(55)$ & $40.0(100)$ \\
\hline
\end{tabular}

Table 2: Unadjusted Odds ratios (OR), 95\% Confidence Intervals (CI) and $P$ values from bivariate logistic regression models $(n=250)$

\begin{tabular}{|l|l|l|l|}
\hline Variables & Unadjusted OR & 95\% CI & P-value \\
\hline Age & & & 0.001 \\
\hline Older adults vs Young adults & 2.41 & $1.45-3.99$ & \\
\hline Gender & & & 0.617 \\
\hline Male vs Female & 0.87 & $0.50-1.51$ & \\
\hline Race & & & 0.929 \\
\hline African American vs White & 0.97 & $0.44-2.12$ & 0.229 \\
African American vs Hispanic & 1.59 & $0.75-3.39$ & 0.783 \\
African American vs Others & 0.83 & $0.22-3.18$ & \\
\hline Marital Status & & & 0.726 \\
\hline Single vs married & 0.80 & $0.29-2.71$ & 0.641 \\
\hline Living Arrangement & & & \\
\hline Homeless vs Domiciled & 0.87 & $0.46-1.64$ & 0.020 \\
\hline IM Medication & & & \\
\hline Yes vs No & 2.08 & $1.12-30.89$ & 0.433 \\
\hline Shift & & & \\
\hline Weekday vs Weekend & 1.25 & $0.71-2.20$ & 0.235 \\
\hline Admission disposition & & & \\
\hline Inpatient unit vs detox & 1.36 & $0.82-2.26$ & \\
\hline
\end{tabular}

Table 3: Adjusted Odds ratios (OR), $95 \%$ Confidence Intervals $(\mathrm{CI})$ and P-values from multivariate logistic regression model $(\mathrm{n}=\mathbf{2 5 0})^{\beta}$

\begin{tabular}{|l|l|l|l|}
\hline Variables & Adjusted OR & 95\% CI & \\
\hline Age & & & \\
\hline
\end{tabular}




\begin{tabular}{|c|c|c|c|}
\hline Older adults vs Young adults & 2.41 & $1.39-4.19$ & 0.002 \\
\hline \multicolumn{4}{|l|}{ Gender } \\
\hline Male vs Female & 1.04 & $0.57-1.88$ & 0.891 \\
\hline \multicolumn{4}{|l|}{ Race } \\
\hline African American vs White & 1.04 & $0.46-2.37$ & 0.913 \\
\hline African American vs Hispanic & 1.61 & $0.72-3.64$ & 0.248 \\
\hline African American vs Others & 1.17 & $0.29-4.77$ & 0.844 \\
\hline \multicolumn{4}{|l|}{ Marital Status } \\
\hline Single vs married & 0.84 & $0.47-1.52$ & 0.575 \\
\hline \multicolumn{4}{|l|}{ Living Arrangement } \\
\hline Homeless vs Domiciled & 0.74 & $0.36-1.51$ & 0.410 \\
\hline \multicolumn{4}{|l|}{ IM Medication } \\
\hline Yes vs No & 1.86 & $0.93-3.74$ & 0.079 \\
\hline \multicolumn{4}{|l|}{ Shift } \\
\hline Weekday vs Weekend & 1.18 & $0.53-2.14$ & 0.584 \\
\hline \multicolumn{4}{|l|}{ Admission disposition } \\
\hline Inpatient unit vs detox & 0.95 & $0.39-1.95$ & 0.857 \\
\hline
\end{tabular}

Table 4: Hosmer-Lemeshow and Pearson Goodness-of-Fit Statistics

\begin{tabular}{|l|l|l|l|}
\hline Criterion & Number of groups or covariate patterns & $\chi 2$ & P-value \\
\hline Hosmer-Lemeshow & 9 & 3.66 & 0.81 \\
\hline Pearson & 95 & 84.06 & 0.47 \\
\hline
\end{tabular}

\section{Discussion}

In this retrospective study, we reviewed the electronic medical records (EMR) of patients with mental health issues or substance use who presented to the psychiatric emergency department (PED) over a one-month period. Out of a total of 493 patients who had presented to the psychiatric ED, 221 patients were discharged home and 22 patients were medically unstable and required further stabilization on the medical floor.

Of the 250 patients who met criteria for psychiatric admission, 150 patients $(60.0 \%)$ were admitted to the inpatient psychiatric unit and 100 patients $(40.0 \%)$ were admitted to the detoxification unit for treatment of substance use disorder. Of the 250 patients, $71.6 \%$ were African Americans, $11.65 \%$ were White, $13.2 \%$ were Hispanic, and $3.6 \%$ were identified as Other Race. The aim of our study is to examine factors responsible for a prolonged length of stay in the psychiatric emergency room. A significant proportion of the psychiatric admissions were patients in an acutely psychotic or manic state with significant risk to self or others and required involuntary admission, however although our study variables did not include diagnosis or weather patient is voluntarily or involuntarily admitted. It is of note that patients admitted for treatment of substance use disorder did so under voluntary status. Both group of patients met the criteria for an admission either to the inpatient psychiatric unit or the inpatient detox program, respectively. The outcome measure of prolonged (LOS) in the PED for our study was a duration of greater than 7.4 hours. In this study, 71 patients $(56.3 \%)$ percent of the psychiatric patients and 55 $(43.7 \%)$ of the detox patients stayed in the psychiatry emergency services (PES) for more than 7.4 hours. Overall, our mean LOS for the inpatient admission was 8.72 hours.

Risk factors for an increased LOS established in our study included patient demographics such as age $>44$ years and pharmacological management (use emergency Intramuscular (IM) medication) of acutely agitated patients. In addition, weekday shift admissions compared with weekend admissions had significant contributions to prolong length of stay in the PED although not found to be statistically significant. There are limited data available on factors associated with prolonged LOS in psychiatry emergency services (PES). A comparative study by Chun-Chi Hsu ${ }^{[8]}$ found that prolonged LOS was associated with use of restraints in the PES, history of illicit substance use and first time visit to the hospital. In our study, we found that a large proportion of patients admitted to the psychiatric inpatient unit had comorbid substance use. Patients admitted for substance use treatment (detox) had to have a history of substance use supported by positive qualitative urine toxicology analysis in order to qualify for admission. However, our result found psychiatric inpatient admission had significant odd for prolonged length of stay in the PED. Our findings also showed a higher odd of prolonged LOS in the PED with being African American compared to being Hispanic, however this was not statistically significant.

Although there is an effect of age $>44$ years with increase in total LOS ( $p$ 0.001). In this study, some studies show that there are no significant age differences that lead to an extended length of stay ${ }^{[13-15]}$. In contrast to previous studies, another study found that age per se was not independently associated with prolonged ED LOS ${ }^{[16]}$. The study suggested that association of increase age and prolong LOS is probably explained by a higher percentage of diagnostic, testing and consultations in older people.

Another retrospective case control study found that extremely long length of stay in ED was due to number of factors such as insurance status (self-pay), transfer to a remote facility, suicidal ideation and the day of arrival of mentally ill patients such as weekday vs. weekends ${ }^{[17]}$. They found that patients who were to visit $\mathrm{ED}$ on weekdays were more likely to be admitted to psychiatric unit than on weekends. On the contrary, this study showed that patients who present to ED on weekday were more likely to have prolonged LOS although not statistically significant $(\mathrm{p}=0.433)$.

A retrospective cross-sectional study found that length of stay is multifactorial: seclusion during admission, accommodation problem and living in an area lacking assertive community services were few factors affecting $\operatorname{LOS}{ }^{[18]}$, homelessness vs having an accommodation had no significant findings in our study. Other variables were not considered in our study. Another study found 
that teaching status of the hospital was not a factor for prolonging LOS. The study rather found that patient presentation with phenomenon such as suicidality and schizophrenia had prolonged length of stay compared to other psychiatric pathologies [19], however our study did not examine association of psychiatric diagnosis with prolonged LOS even although most of the psychopathological cases that present to our PED presented with diagnoses are schizophrenia spectrum disorders. Certain race and age groups such as African Americans, Hispanics, and age $>45$ years were associated with prolong LOS in a study by Smith et al ${ }^{[19]}$. Study result shows that age $>44$ and being African American are associated with prolong LOS. Transfer of patients to other facility have also been reported to significantly contribute to prolonged LOS ${ }^{[17,20,21]}$, although this is not a variable considered in our study.

Some of the limitations of this study include its retrospective nature, unable to discern which diagnosis has more input to prolong LOS, unable to determine if comorbid substance use contributed to prolonged LOS for Inpatient disposition compared to detox, unable to ascertain if impatient bed availability and insurance status contributed to prolonged LOS.

Future directions will include providing intervention to tackle areas with significant statistical result with aim of reducing the total LOS. These interventions may include staffing method, making sure medicated patients are immediately admitted to the inpatient units and can be reviewed by the medical team on the unit once patient is alert. The research team will compare the results of this study post implementation of recommended intervention.

\section{Conclusion}

In conclusion, prolonged LOS was common in this study; internal and external factors were identified. Some of these factors include patient characteristics, time of patient arrival (shift schedule patient arrived) and whether patient is medicated for psychotic agitation or aggression. Understanding the factors that contribute to prolonged LOS in the PED is a critical step in improving ED patient care efficiency. Therefore, measures should be put in place to reduce patients LOS in the PED thereby preventing overcrowding.

\section{Acknowledgments}

The authors wish to thank Dr. Noela Co, Director of Quality Assurance, Department of Psychiatry, Interfaith Medical Center, Brooklyn, New York, for her support and assistance.

\section{Financial Disclosure}

The authors have no financial disclosure.

\section{Conflict of Interest}

The authors declare no conflict of interest.

\section{Informed Consent}

Not applicable.

\section{Data Availability}

The data supporting the findings of this study are available from the corresponding author upon reasonable request.

\section{Author Contributions}

Chiedozie Ojimba contributed to the design of the study, data collection, interpretation of the data, helped to write the final draft of the manuscript, and has full access to all the data in the study as the primary author of the manuscript. AI contributed to the design of the study, data collection and helped to write the final draft of the manuscript. OJ contributed to the design of the study and helped with data analysis and interpretation. TT, SA, OO, AM, SK contributed to the design of the study, data collection and helped to write the manuscript. AS, $\mathrm{KC}, \mathrm{AO}$ contributed to the design of the study and helped with data collection. TO contributed to the study design and supervised the writing of the final draft of the manuscript. JH contributed to the design of the study and supervised the writing of the final draft of the manuscript.

\section{References}

[1] Owens PL, Mutter R, Stocks C. Mental Health and Substance Abuse-Related Emergency Department Visits Among Adults, 2007. HCUP Statistical Brief \#92. July 2010. U.S. Agency for Healthcare Research and Quality, Rockville, MD

[2] Agency for Healthcare Research and Quality. Chartbook on Care Coordination. Measures of Care Coordination: Preventable Emergency Department Visits. May 2015. Rockville, MD: Agency for Healthcare Research and Quality

http://www.ahrq.gov/research/findings/nhqrdr/2014chart books/carecoordination/careco ord-measures2.html. Accessed June 28, 2016.

[3] Weiss AJ (Truven Health Analytics), Barrett ML (M.L. Barrett, Inc.), Heslin KC (AHRQ), Stocks C (AHRQ). Trends in Emergency Department Visits Involving Mental and Substance Use Disorders, 2006-2013. HCUP Statistical Brief \#216. December 2016. Agency for Healthcare Research and Quality, Rockville, MD.

[4] Yoon, P., Steiner, I., \& Reinhardt, G. (2003). Analysis of factors influencing length of stay in the emergency department. Canadian Journal of Emergency Medicine, 5(3), 155-161.

[5] Schneider, S. M., \& Winograd, S. M. (2009). Emergency department crowding. Emergency Medicine Reports, 30(3), 13-23.

[6] Zeller, S., Calma, N., \& Stone, A. (2014). Effects of a dedicated regional psychiatric emergency service onboarding of psychiatric patients in area emergency departments. Western Journal of Emergency Medicine, 15(1), 1.

[7] Pearlmutter, M. D., Dwyer, K. H., Burke, L. G., Rathlev, N., Maranda, L., \& Volturo, G. (2017). Analysis of emergency department length of stay for mental health patients at ten Massachusetts emergency departments. Annals of emergency medicine, 70(2), 193-202.

[8] Hsu, C. C., \& Chan, H. Y. (2018). Factors associated with prolonged length of stay in the psychiatric emergency service. PloS one, 13(8), e0202569.

[9] Lippert, S. C., Nesper, A., Jain, N., Fahimi, J., Pirrotta, E., \& Wang, N. E. (2016). 142 Mental health emergency department visits: 24 hours and counting, characteristics associated with prolonged length of stay. Annals of Emergency Medicine, 68(4), S57. 
[10] American College of Emergency Physicians. ACEP Psychiatric and Substance Abuse Survey. Irving, TX: American College of Emergency Physicians; 2008.

[11] Singer, A. J., Thode Jr, H. C., Viccellio, P., \& Pines, J. M. (2011). The association between length of emergency department boarding and mortality. Academic Emergency Medicine, 18(12), 1324-1329.

[12] Liew, D., Liew, D., \& Kennedy, M. P. (2003). Emergency department length of stay independently predicts excess inpatient length of stay. Medical Journal of Australia, 179(10), 524-526.

[13] Kreindler SA, Cui Y, Metge CJ, Raynard M. Patient characteristics associated with longer emergency department stay: a rapid review. Emerg Med J. 2016; 33: 194-9.

[14] Chaou CH, Chen HH, Chang SH, et al. Predicting length of stay among patients discharged from the emergency department-using an accelerated failure time model. PLoS One. 2017; 12: e0165756.

[15] Vegting IL, Alam N, Ghanes K, et al. What are we waiting for? Factors influencing completion times in an academic and peripheral emergency department. Neth $\mathbf{J}$ Med. 2015; 73: 331-40.

[16] Van der Veen et al (2018). Independent determinants of prolonged emergency department length of stay in a tertiary care center: a prospective cohort study.
Scandinavian Journal of Trauma, Resuscitation and Emergency Medicine (2018) 26:81

[17] Stephens, R.J., White, S.E., Cudnik, M., Patterson, E.S. (2014). Factors associated with Longer Length of Stay for Mental Health Emergency Department Patients. Journal of Emergency Medicine, Volume 47, Issue 4, 412-419.

[18] Zhang, J., Harvey, C., Andrew, C. (2011). Factors associated with length of stay and the risk of readmission in an acute psychiatric inpatient facility: a retrospective study. Australian and New Zealand Journal of Psychiatry; 45: 578-585

[19] Smith, JL., De Nadai, AS., Storch, EA. Langland-Orban, B., Pracht, E., Petrila, J. (2016). Correlates of length of stay and boarding in florida emergency departments for patients with psychiatric diagnoses. Psychiatr Serv. 2016 November 01; 67 (11): 1169-1174

[20] Chang G, Weiss AP, Orav EJ, et al.: Hospital variability in emergency department length of stay for adult patients receiving psychiatric consultation: a prospective study. Annals of emergency medicine 58:127-36 e1, 2011.

[21] Weiss AP, Chang G, Rauch SL, et al.: Patient- and practice-related determinants of emergency department length of stay for patients with psychiatric illness. Annals of emergency medicine 60:162-71 e5, 2012. 